\title{
A Network of HSPG Core Proteins and HS Modifying Enzymes Regulates Netrin-Dependent Guidance of D- Type Motor Neurons in Caenorhabditis elegans
}

\author{
Stephan Gysi ${ }^{1,2}$, Christa Rhiner ${ }^{3}$, Stephane Flibotte ${ }^{4}$, Donald G. Moerman ${ }^{4}$, Michael O. Hengartner ${ }^{1,2^{*}}$ \\ 1 Institute of Molecular Life Sciences, University of Zurich, Zurich, Switzerland, 2 Neuroscience Center Zurich, University of Zurich, Zurich, Switzerland, \\ 3 Institute of Cell Biology, University of Bern, Bern, Switzerland, 4 Department of Zoology, University of British Columbia, Vancouver, Canada
}

\begin{abstract}
Heparan sulfate proteoglycans (HSPGs) are proteins with long covalently attached sugar side chains of the heparan sulfate (HS) type. Depending on the cellular context HS chains carry multiple structural modifications such as sulfate residues or epimerized sugars allowing them to bind to a wide range of molecules. HSPGs have been found to play extremely diverse roles in animal development and were shown to interact with certain axon guidance molecules. In this study we describe the role of the Caenorhabditis elegans HSPG core proteins Syndecan (SDN-1) and Glypican (LON-2) and the HS modifying enzymes in the dorsal guidance of D-type motor axons, a process controlled mainly by the conserved axon guidance molecule UNC-6/Netrin. Our genetic analysis established the specific HS code relevant for this axon guidance event. Using two sensitized genetic backgrounds, we isolated novel components influencing D-type motor axon guidance with a link to HSPGs, as well as new alleles of several previously characterized axon guidance genes. Interestingly, the dorsal axon guidance defects induced by mutations in zfp-1 or lin-35 depended on the transgene ox/s12 used to visualize the D-type motor neurons. ox/s12 is a large multi-copy transgene that enlarges the $X$ chromosome by approximately $20 \%$. In a search for genes with a comparable phenotype we found that a mutation in the known dosage compensation gene $d p y-21$ showed similar axon guidance defects as zfp-1 or lin-35 mutants. Thus, derepression of genes on X, where many genes relevant for HS dependent axon guidance are located, might also influence axon guidance of $\mathrm{D}$-type motor neurons.
\end{abstract}

Citation: Gysi S, Rhiner C, Flibotte S, Moerman DG, Hengartner MO (2013) A Network of HSPG Core Proteins and HS Modifying Enzymes Regulates Netrin-Dependent Guidance of D-Type Motor Neurons in Caenorhabditis elegans. PLoS ONE 8(9): e74908. doi:10.1371/journal.pone.0074908

Editor: Brian D. McCabe, Columbia University, United States of America

Received March 5, 2013; Accepted August 7, 2013; Published September 16, 2013

Copyright: $\odot 2013$ Gysi et al. This is an open-access article distributed under the terms of the Creative Commons Attribution License, which permits unrestricted use, distribution, and reproduction in any medium, provided the original author and source are credited.

Funding: This work was supported by the Neuroscience Center Zurich (www.neuroscience.ethz.ch), the Kanton of Zurich, the Swiss National Science Foundation (www.snf.ch), and the Ernst Hadorn Foundation. The funders had no role in study design, data collection and analysis, decision to publish, or preparation of the manuscript.

Competing interests: The authors have declared that no competing interests exist.

*E-mail: michael.hengartner@imls.uzh.ch

\section{Introduction}

Extension and guidance of axons during nervous system development rely on a number of conserved axon guidance cues [1]. In addition, there is increasing evidence that other systems fine-tune the known major axon guidance cue families. Heparan Sulfate Proteoglycans (HSPGs) have been shown to encode specific information for the development of nervous systems through specifically modified covalently attached carbohydrate polymers, the glycosaminoglycan (GAG) heparan sulfate (HS) $[2,3,4,5,6]$. The core proteins can be grouped into two classes, (i) membrane associated core proteins, such as the transmembrane Syndecans ( $s d n-1$ in C. elegans) and the glycosylphosphoinositol (GPI) anchored Glypicans (gpn-1, lon-2) and (ii) core proteins of the extra cellular matrix (ECM), which include Perlecan (unc-52), Agrin (agr-1) and Collagen XVIII (cle-1) (reviewed in 7,8,9). Studies in various organisms including worms, flies and mammals have shown that HSPGs play diverse roles in the nervous system (reviewed in 10). However, studying HSPGs in mammals is challenging because most members are present in multiple copies and are most probably functionally redundant.

While the core proteins for HS chains are responsible for some diversity of HSPGs in different cell types, the largest source of diversity comes from variable modifications of heparan-sulfate disaccharides, including the epimerization of the fifth carbon atom in hexuronic acid and sulfation at several sites. Similar to the core proteins, the genes coding for the enzymes catalyzing the modifications are well conserved in $C$. elegans [11]. Deacetylation is performed by $h s t-1$, which is an essential gene in the worm, probably due to the fact that deacetylation precedes and paves the way for all sulfations. HS chains in hst-1 mutants are most likely entirely unsulfated and therefore lack any specificity. 2-O, 3-O, and 6-O sulfations are 
catalyzed by hst-2, hst-3.1 and hst-3.2, and hst-6, respectively. The gene for C5 epimerization is hse-5. Finally, the HS chains are about 100 or more sugar units long and have numerous structural heterogeneities offering the possibility to interact with a large number of ligands [12,13,14,15]. The large negative charge of the HS chains is a result of sulfation and is one reason why many molecules bind HS. The modifications are complex and incomplete, giving rise to an enormous number of possible modification patterns [16,17]. A core protein can therefore carry different context dependent modifications, resulting in an HS code that is required on a specific neuron or in its environment to modulate certain axon guidance choices $[9,16,18]$.

Across all species, populations of axons project towards or away from the midline of the nervous system. Members of the highly conserved Netrin family of proteins mainly mediate this attraction and repulsion. The Netrins are secreted guidance molecules with one known member in C. elegans (UNC-6 [19]). Also, the receptor mechanisms for Netrin are highly conserved throughout the animal kingdom. Growth cone attraction is triggered by the binding of UNC-6/Netrin to homodimers of UNC-40/deleted in colorectal cancer (DCC) receptor family proteins, while growth cone repulsion is mediated by the UNC-5 family of receptor proteins, in some cases together with UNC-40 [20], (reviewed by [21]). In the worm, UNC-5, UNC-6 and UNC-40 represent the major system for circumferential guidance of migrating cells and axons towards and away from the ventral nerve cord (VNC) [19].

The major classes of motor neurons in C. elegans (DA, DB, $\mathrm{DD}, \mathrm{VD}$ and AS motor neurons) have cell bodies in the VNC and send commissural axons to the dorsal nerve cord (DNC). UNC-6 is expressed ventrally to form a gradient. UNC-5 and UNC-40 are expressed in dorsally migrating axons to mediate a repulsive response to UNC-6, whereas UNC-40 is expressed in ventrally migrating axons to mediate an attractive response to UNC-6 [22,23].

In addition to UNC-6 the dorsally expressed UNC-129/TGF- $\beta$ family ligand that establishes a gradient opposite to UNC-6 also plays an important role in guidance of dorsally migrating axons [24]. Axons growing out from the ventral side of the worm first use the UNC-5 receptor to respond to high concentrations of UNC-6. As the growth cone moves further away from the ventral side the concentration of UNC-6 decreases while the concentration of UNC-129 increases. UNC-129 binds to UNC-5 and is thought to induce a switch in UNC-6 signaling from the UNC-5 only signaling to UNC-5 + UNC-40 signaling, which is more sensitive to UNC-6 and can therefore maintain the repulsive effect of UNC-6 even in areas where the UNC-6 concentration is very low [24]. In Drosophila the establishment of the Dpp gradient, a TGF- $\beta$ homolog was shown to depend on HSPGs [25]. What role HSPGs play in the integration of the UNC-6 and UNC-129 gradients in C. elegans is an open question.

In mice, axons growing towards the floor plate in the spinal chord fail to respond to the attractive Netrin signal secreted by the floor plate if HSPGs lack functional HS side chains [26], suggesting that HSPG function is vital for proper Netrin signaling. We therefore hypothesized that a systematic study of the HSPG network required for ventral to dorsal axon guidance in $C$. elegans would provide the possibility to establish a clearer link between Netrin and HSPGs and to define the HS code relevant for Netrin signaling. In this study we show that the HSPG network guiding D-type motor axons is comprised of two parallel pathways containing either SDN-1 or LON-2 as core protein. In an enhancer screen we find new components influencing D-type motor axon guidance and show that Netrin signaling through the UNC-5 receptor could depend on HSPGs. Furthermore, our data indicate that derepression of genes located on the $X$ chromosome influences axon guidance, most likely because many genes relevant for D-type motor axon guidance are located on $\mathrm{X}$.

\section{Materials and Methods}

\section{Strains, general handling and procedures}

Standard methods were used for maintenance and handling of $C$. elegans as described by 27 . Briefly, worms were grown on E. coli (strain OP50) and the C. elegans variety Bristol N2 was used as wild type and kept at $20^{\circ}$ if not stated otherwise. The Hawaii variety (CB4856) was used for mapping of mutations.

The following mutations and transgenes were used:

LGI: lin-35(n745) [28], oxls268[unc-47::gfp] [29], cle-1(cg120) [30], unc-73(op464) (this study).

LGII: ptp-3(op147) [31], ptp-3(ok244), unc-52(e444) [32], unc-53(ok2736), unc-53(op479) (this study), unc-130(oy10) [33], unc-130(op459) (this study), juls76[unc-25::gfp; lin-15(+)] [34], hst-3.1(ok1249) [35], hst-3.1(tm734) [35].

LGIII: hse-5(tm472) [16], zfp-1(ok554) [36], zfp-1(op481) (this study).

LGIV: eri-1(mg366) [37], unc-129(ok1443), unc-5(e53) [27], unc-5(op460), unc-5(op468), unc-5(op469), unc-5(op477) (all this study), zag-1(op462) (this study).

LGV: oyls14[sra-6::gfp; lin-15(+)] (Piali Sengupta), max-1(op482) (this study), dpy-21(e428) [38].

LGX: lon-2(e678) [27], hst-6(ok273) [16], sdn-1(zh20) [5], hst-2(ok595) [16], gpn-1(tm588) [39], ox/s12[unc-47::gfp; lin-15(+)] [40], hst-3.2(tm3208) [35], hst-3.2(tm3006) [35], pkls296[hsp::gsa-1(Q208L); dpy-20(+)] [41].

\section{Ethyl Methanesulfonate (EMS) Enhancer screens}

For mutagenesis, staged hse-5(tm472) or sdn-1(zh20) mutant L4 animals carrying the oxls12 transgene were mutagenized with ethyl methanesulfonate (EMS) as described by 27 and distributed onto four $9 \mathrm{~cm}$ seeded plates ( 3 P0/plate). These P0 animals were kept at $15^{\circ} \mathrm{C}$ and transferred to new plates twice a day, until they stopped laying eggs. The F1 animals were grown at $20^{\circ} \mathrm{C}$ and allowed to lay eggs for about $12 \mathrm{~h}$ before they were counted to estimate the number of haploid genomes screened and removed. Adult F2 animals were screened for candidates with severely defective D-type motor neurons under a dissection fluorescence microscope.

Mutants (generated in the Bristol N2 background) were crossed to animals of the Hawaii (CB4856) background and the mutations mapped using fragment length polymorphism (FLP) mapping as described by 42 . Following FLP mapping, we used 
three distinct strategies to identify candidate genes. In some cases, after sampling about 200-300 recombinants, interval sizes could be narrowed to containing 100-300 genes, which were subsequently treated with RNAi by feeding to identify possible candidate genes. Alternatively, mutations were mapped to intervals of roughly $1 \mathrm{Mb}$ in size and then subjected to array CGH to find the mutation as described by 43,44 . As a third means to find mutations, we made use of the whole genome sequencing strategy described by 45 . FLP mapping was needed to map candidates to roughly $4 \mathrm{Mb}$ intervals. Genomic DNA was sequenced on an Illumina sequencer and the sequencing reads aligned to the N2 reference sequence with the MAQGene software [46]. The sequencing data can be accessed via the following URLs: For strain WS3835 containing op462: http://www.ebi.ac.uk/ena/data/view/ PRJEB4267; for strain WS3855 containing op472: http:// www.ebi.ac.uk/ena/data/view/PRJEB4268; for strain WS4018 containing op476: http://www.ebi.ac.uk/ena/data/view/ PRJEB4269.

\section{RNAi}

All RNAi experiments were done in strains carrying the eri-1(mg366) mutation to render worms sensitive to RNAi [37]. For detection of candidate genes from the screen the strains subjected to RNAi additionally carried the background mutation used during the screen (either sdn-1(zh20) or hse-5(tm472)) plus the oxls12 transgene. For all other RNAi experiments the strain eri-1(mg366); sdn-1(zh20) oxls12 was used. RNAi bacteria were seeded on nematode growth medium (NGM) agarose plates containing $1 \mathrm{mM}$ Ampicilin and 2mM IPTG. Worms were kept on RNAi bacteria for at least two generations before D-type motor axon guidance phenotypes were assessed.

\section{Scoring of D-type axon guidance defects}

The D-type motor neurons were visualized with the oxls12[unc-47::gfp; lin-15(+)], ox/s268[unc-47::gfp] or juls76[unc-25::gfp; lin-15(+)] transgenes. Two different decision points during axon outgrowth were scored in L1 animals: (i) the number of commissural axons turning away from the VNC (commissures/L1) and (ii) the number of commissural axons reaching the DNC (commissures reaching dorsal nerve cord). Fifty animals were scored for every genotype. Differences between pairs of strains regarding the number of commissures reaching the DNC were tested for statistical significance with a Mann-Whitney $U$ test for non-parametric data using the freely available software $R$ ( $R$ core team 2013) [47]. Double mutants were always compared to the corresponding single mutant with the stronger defect.

\section{Detection of integration site of oxIs12}

The WGS data from all the three sequenced strains containing ox/s12 (originally sequenced for mutation detection) were pooled and aligned to the $C$. elegans reference sequence using the CLC Genomic Workbench (www.clcbio.com) with standard alignment settings given by CLC. All the aligned reads were discarded. The non-aligned reads were aligned a second time to the $C$. elegans reference sequence, this time allowing for up to 56 misaligned bases per 76 base read. Therefore the vast majority of the reference genome remained uncovered but reads that spanned the break points of the transgene and remained unaligned after the first alignment because they contained mainly transgene sequence were aligned at the break points in this second round. Manual inspection of an interval of +/- 1cM from sdn-1, where we expected the integration site based on recombination frequencies, revealed two accumulations of reads with sharp break points. A blast alignment of the up to 50 non-aligned bases of the sequencing reads revealed good similarities to plasmid backbone sequences that would clearly not be found in wild type $C$. elegans.

\section{Determination of transgene size from WGS data}

During the first alignment of the sequencing reads, all the reads originating from the transgene with endogenous counterparts will align at that endogenous position. Therefore analyzing the coverage in these areas should allow us to estimate the number of copies of the corresponding fragment present in the transgene. Figure $\mathrm{S} 1$ shows the coverage across the lin-15AB locus. While the average coverage on chromosome $X$ (i.e. where lin-15AB are located) is $76.23 \mathrm{x}$, it is dramatically increased at the lin- $15 A B$ locus to $11^{\prime} 030 x$, suggesting the presence of about 140 copies of the lin-15AB(+) construct. The part of the lin-15AB locus contained in the transgene (10'970bp) can be determined precisely since coverage increases from around $50 x$ to over 4 '000x at the first base included in the transgene. Using the same estimation procedure, there are roughly 170 copies of unc- 47 spanning $5180 \mathrm{bp}$ plus 130 copies of the unc-54 3' UTR with a length of $411 \mathrm{bp}$. The fact that we find 170 copies of unc- 47 and only 130 copies of the unc-54 3' UTR, which are both a part of the unc-47::gfp construct, reflects the highly variable coverage also seen at the lin-15AB locus (Figure S1) and strongly suggests that the majority of the copies are neither complete nor functional. Additionally, we estimate the presence of about 150 copies of gfp sequence with a length of $867 \mathrm{bp}$ plus about 310 plasmid backbone copies of $\approx 3$ '000bp. Taken together this suggests that $o x / s 12$ has a total size of about $3.6 \mathrm{Mb}$.

\section{qPCR for transgene size estimations}

Genomic DNA was prepared form mixed stage worms of either N2 wild type, oxls12 or pkls296 animals. In ox/s12 animals lin-15AB and unc-47 were tested and in pkls296 $d p y-20$ and gsa-1. The following primers were used:

\section{lin-15AB: fw: CCGAGAAGTGAGGAACGTTT rv: ATGGCTTCGGCAAATTAGGT unc-47: fw: ATTGGCTCTGATGCTTCTCC rv: AGCAACCACAAAAAGACGAC dpy-20: fw: TAGAGGCCATGTCTGAGGAT rv: GGAATGCAACAAGACCCAAC gsa-1: fw: CCCTGGCCCTATTCAAAAAC rv: CGCTTGGCCTTGATTTTCTC}

The average cycle number difference $(\Delta \mathrm{ct})$ of three independent experiments between $\mathrm{N} 2$ and the transgenic 
strains was determined and the copy numbers of the different constructs calculated as $2^{\Delta \mathrm{ct}}$.

In the case of ox/s12 this approach led to the detection of about 120 copies of lin-15AB and 130 copies of unc-47, leading to a size estimation for oxls12 of about $2.9 \mathrm{Mb}$. For pkls296, qPCR indicated the presence of 14 copies of the gsa-1 construct, which is about $9.5 \mathrm{~kb}$ long and 65 copies of $d p y-20$ (about $9.1 \mathrm{~kb}$ ) leading to a size estimation of $0.72 \mathrm{Mb}$. Additionally, pkls296 contains an unknown amount of empty vectors [41], most likely leading to an increase in size by about two to three folds indicating that pkls296 is probably slightly smaller in size compared to oxls12.

\section{Results}

\section{A network of HSPG core proteins and HS modifying enzymes controls guidance of D-type motor neurons}

During the study of the function of single HSPG core proteins and modifying enzymes in $C$. elegans it has been noted that single mutants, depending on the context, displayed specific defects in axon guidance as well as cell migration $[5,16]$. However, overall the defects found in these mutants were relatively weak, in particular in the guidance of D-type motor neurons (for representative pictures see Figure 1B-E). We hypothesized that the weak defects in axon guidance might be due to the presence of multiple core proteins acting in parallel. To test this, we systematically analyzed D-type motor axon guidance in double and triple mutants of the HSPG core proteins sdn-1/Syndecan, Ion-2/Glypican, gpn-1/Glypican, cle-1/Collagen XVIII and unc-52/Perlecan. We observed a significant increase in guidance defects in lon-2(e678) $s d n-1(z h 20)$ double mutants, suggesting that SDN-1 and LON-2 act in parallel genetic pathways to promote D-type motor axon guidance (Figure 1A and S2). In contrast, we found no role in this process for GPN-1, the second Glypican homolog of $C$. elegans, which is in agreement with our prior observations [5]. Although the cle-1(cg120) single mutant had a weak D-type axon guidance defect, the loss of cle-1 failed to significantly enhance either sdn-1 or lon-2 mutants, suggesting an indirect effect of the loss of CLE-1 function on D-type axon guidance (Figure S2). It should be noted that while cle-1(cg120) is a strong loss of function mutation it is not a null allele [48]. Residual cle-1 function could therefore interfere with our double/triple mutant analysis. UNC-52/Perlecan has been described to play a role in Netrin signaling during ventral to dorsal migration of the distal tip cell of the $C$. elegans gonad [49]. However, our analysis of different unc-52 alleles indicated that unc-52 has no function in guidance of D-type motor neurons (unc-52(e444): Figure S2, data for alleles e669, e1421, and $g k 3$ not shown). Since unc-52 null alleles are lethal only hypomorphic alleles were used. We also tested mutations in agr-1/Agrin and VC5.2, a gene with similarity to Perlecan but found that these core proteins are not involved in D-type motor axon guidance (data not shown).

Earlier work indicated the importance of cell type-specific modifications of the HS chains by various HS modifying enzymes [17]. Different modifications can confer distinct function to a common HSPG core protein, which led to the proposal of an HS code for HSPGs [16]. We therefore built double and triple mutants of genes coding for a core protein and one of the HS sulfotransferases hst-2, hst-3.1, hst-3.2, hst-6 or the C5-epimerase hse-5 with the aim to find the HS code for SDN-1 and LON-2 for their function in dorsal guidance of D-type motor neurons. Mutations in hst-2 and hse-5 strongly enhanced D-type axon guidance defects of the $s d n-1(z h 20)$ mutant but resulted in no enhancement when combined with lon-2(e678) (Figure 1A and S2). This suggests that HST-2 and HSE-5 might act mainly on LON-2. This is compatible with the finding that lon-2, hst-2, and $h s e-5$ are expressed in the hypodermis $[16,50,51]$ while $s d n-1$ and hst- 6 expression is found mainly in neurons $[5,16]$.

There are two $C$. elegans genes coding for a protein with potential HS-3-O sulfotransferase activity, hst-3.1 and hst-3.2. The hst-3.1; hst-3.2 sdn-1 as well as the hst-3.1; hst-3.2 lon-2 triple mutants failed to further increase D-type motor axon guidance defects, indicating that HS-3-O sulfotransferase activity is not important for this axon guidance event (Figure $1 \mathrm{~A}$ and S2).

A particular situation was found with hst-6. A mutation in this gene clearly enhances $s d n-1(z h 20)$ while the lon-2(e678) hst-6(ok273) double mutant shows little defect (Figure S2), indicating that HST-6 acts on LON-2 and in parallel to SDN-1. However, both the hst- 6 hst- 2 and $h s e-5$; hst- 6 double mutants do show increased defects (Figure 1A) indicating that HST-6, besides acting on LON-2, modifies the HS chains of an additional core protein. Because hst-6(ok273) does not further enhance the lon-2 sdn-1 double mutant (Figure 1A), it is likely that this additional HSPG core protein is in fact SDN-1, rather than another uncharacterized HSPG. Taken together, our data show that SDN-1 and LON-2 are the two HSPG core proteins relevant for $D$-type motor axon guidance. We were also able to determine the HS code of the sugar side chains of these two core proteins: HSE-5 and HST-2 act mainly on LON-2 in D-type dorsal guidance, whereas modification by HST-6 is required both on SDN-1 and LON-2. We propose that the resulting modified HS chains on LON-2 and SDN-1 have the affinity to bind to certain as yet unknown axon guidance molecules.

\section{A screen for genes acting upstream or downstream of HSPGs during D-type motor axon guidance}

From our mutational analysis of HSPG core proteins and HS modifying enzymes we concluded that HSPGs are involved in at least two pathways influencing D-type motor axon guidance, one involving SDN-1 the second one HSE-5 and HST-2, acting on LON-2. We decided to use this system in a forward genetic enhancer screen in order to identify additional genes acting in these two pathways (Figure S3). Of all the tested double mutant combinations, the hse-5(tm472); sdn-1(zh20) animals displayed the most severe defects. Therefore we separately mutagenized sdn-1(zh20) oxls12 and hse-5(tm472); oxls12 animals and screened the F2 generation for candidates phenocopying the $h s e-5$; sdn-1 double mutant phenotype. The transgene oxls12 was used to label the D-type motor neurons with gfp. We screened approximately 11'000 genomes for each of the two approaches. Isolated candidates were mapped using fragment length polymorphisms (FLPs) as described by 42 
A $\quad$ commissures/L1 $\quad$ commissures reaching dorsal nerve cord
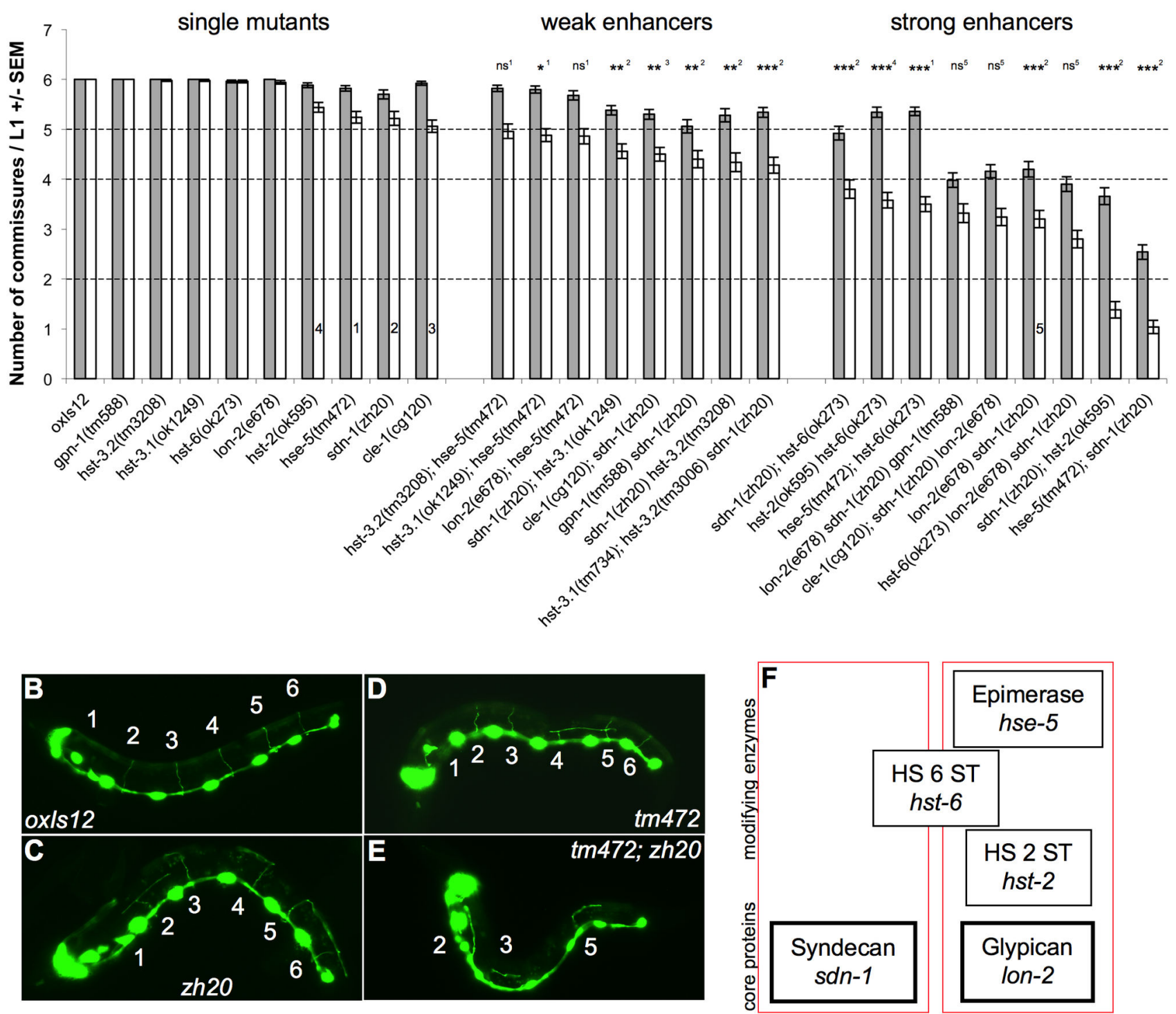

Figure 1. An HSPG network influences D-type motor axon guidance. A: Summary of relevant single and double mutants. All the single mutants have no or only minor defects, meaning that on average no more than one commissural axon fails to reach the dorsal nerve cord (DNC). The dashed line at 5 commissures per animal indicates the limit separating the single mutants from the weak enhancers. Weak enhancers (one to two commissural axons fail to reach the DNC, limit indicated by dashed line at 4 commissures per animal) are double mutants that are considered not to play an important role in D-type axon guidance. Strong enhancers have very clear D-type axon guidance defects (the dashed line at 2 commissures per animal is to allow for distant bars to be visually comparable). Statistical test results are indicated as follows: $n s=$ not significant, ${ }^{*}=p<0.05,{ }^{* *}=p<0.005,{ }^{* * *}=$ $p<0.0005$. Superscripts (also shown in the bars of the corresponding strains) indicate to which strain the comparison was made: 1 : hse-5(tm472), 2: sdn-1(zh20), 3: cle-1(cg120), 4: hst-2(ok595), 5: lon-2(e678) sdn-1(zh20). B-E: Representative pictures of wild type (B), sdn-1(zh20) (C) and hse-5(tm472) (D) single mutants and the strongest enhancer hse-5(tm472); sdn-1(zh20) (E). The transgene ox/s12 is present in all backgrounds. F: HSPG core proteins and HS modifying enzymes can be placed into two groups based on the strength of the D-type motor axon guidance defects observed in double mutants. Only the double mutants of the group of strong enhancers are considered for this network. For a complete set of strains see Figure S2. Grey bars in A represent the number of commissural axons growing away from the ventral nerve cord (VNC); white bars indicate the number of commissural axons reaching the DNC. Numbers are from 50 L1 animals +/- SEM.

doi: 10.1371/journal.pone.0074908.g001 
Table 1. Summary of $s d n-1(z h 20)$ and hse-5(tm472) enhancers.

\begin{tabular}{|c|c|c|c|}
\hline Allele & Gene & Amino acid change & Genomic environment \\
\hline op459 & unc-130 & R219K & CAATGGATCGTTTCTGCGCAAAAGGAAGAGATACAAGAAAA \\
\hline op460 & unc-5 & $\mathrm{C} 25 \mathrm{Y}$ & TAAGCCATTGCGTCTTCAATACCGAGCCAATCACGCAACAA \\
\hline op462 & zag-1 & Q485stop & AGGGTCTCTTCTCGTGCGATTAATGTGACAAGGTGTTCGGC \\
\hline op464 & unc-73 & intron & TATTTGAAGTTACTTCGTAAATTTTTTATTTGACTGAAATG \\
\hline \multirow[t]{2}{*}{ op468 } & unc-5 & S559stop $^{a}$ & 5' flanking region: АССТСАССТСАAАCСAATCG \\
\hline & & & 3' flanking region: AGAGTAATTGTCTATCAAAA \\
\hline op469 & unc-5 & G254E & TCCAGCTCCATTGAACGACGAAGAGTACTGTAAGGGAGAAG \\
\hline op472 & nd & - & - \\
\hline op474 & nd & - & - \\
\hline op476 & nd & - & - \\
\hline op477 & unc-5 & G259R & ACGACGGAGAGTACTGTAAGAGAGAAGAAGAAATGACGAGA \\
\hline op479 & unc-53 & R1258stop & TTACTTCСTCAACTACAATCTGAATGTTCATGCACGGTGCC \\
\hline op480 & nd & - & - \\
\hline op481 & $z f p-1$ & W601stop & ACAGCTGTTGGAACGACAATAGGATCAAGGATCAAGTCTTC \\
\hline op482 & $\max -1$ & W878stop & CTTCGAATGAGACATTATTGAGGCCATTTGGCACTGACAGA \\
\hline
\end{tabular}

either until the phenotypically relevant mutation was found or until intervals were small enough to use arrayCGH [44] or whole genome sequencing [45] for detection of the mutation. We isolated a total of 14 candidates from the two screens and for all but four of these the relevant mutation could be identified (Table 1). Many of the genes detected are related to the Netrin pathway (e.g. unc-5, unc-130, max-1), consistent with the fact that UNC-6/Netrin is the major axon guidance cue responsible for circumferential guidance of D-type motor axons [19].

Of the four unc-5 alleles recovered in the screen, only op468 has clear D-type axon guidance defects as a single mutant (Figure 2A). The op468 allele carries a deletion of $337 \mathrm{bp}$ resulting in a frame shift and a premature termination of translation. Nevertheless, op468 seems to be a hypomorphic allele judged by the fact that the reference allele unc-5(e53) showed more pronounced defects, both on its own and in combination with hse-5(tm472) (Figure 2A). The other unc-5 mutations op460, op469, and op477 are very weak missense point mutations affecting the extra cellular domain indicating that the amino acid sequence of UNC-5 is highly sensitive to changes in the HSPG sensitized background, hinting at a possible interaction between UNC-5 and HS chains on SDN-1 or LON-2 or both.

The TGF $\beta$ ligand UNC-129 and its transcription factor UNC-130 have been described to act in circumferential axon guidance [33] and participate in Netrin signaling [24]. The unc-130 allele recovered from our screen affects the neighboring amino acid mutated in the oy10 reference allele within the conserved forkhead domain. Mutations in unc-129 and unc-130 lead to comparable defects in D-type motor axon guidance (Figure 2A). With max-1 we also found a gene that is thought to act downstream of unc-6/Netrin [34].

We also identified mutations in a number of genes whose link to the Netrin pathway is less clear. UNC-53, like its three human homologs the Navigators NAV1-3, is important for cytoskeletal rearrangements to control cell migration and axon guidance (reviewed in 52). UNC-53 is known to play a role in longitudinal axon guidance and cell migration in $C$. elegans [53]. We found that both the allele from our screen, unc-53(op479), which carries a nonsense mutation and the deletion allele unc-53(ok2736) clearly enhance the $s d n-1(z h 20)$ mutation (Figure 2B), demonstrating that UNC-53 also influences circumferential axon guidance events.

During our search for the gene affected by the op479 mutation, we found that the RNAi clone for ptp-3 gave a positive phenotype. While sequencing revealed that there was no mutation in ptp-3 in candidate op479, the Tc1 transposon insertion allele ptp-3(op147) isolated earlier in our lab led to a clear increase of D-type motor axon guidance defects in the hse-5 or sdn-1 mutant background. It has been reported that op147 is temperature-sensitive [31] and indeed growing ptp-3(op147); sdn-1(zh20) animals at $25^{\circ}$ increases their defects while ptp-3(op147); hse-5(tm472) animals do not survive at $25^{\circ}$ (Figure 2B). PTP-3 is a receptor tyrosine phosphatase of the LAR (Leukocyte Common Antigen related) type. Its homolog in Drosophila has been shown to interact with HS chains on Syndecan and Dallylike/Glypican during the process of synapse formation [54,55]. In order to explore the relevance of LAR signaling during D-type axon guidance in more detail, we tested other LAR signaling components. NID-1/ Nidogen is an extracellular binding partner of LAR and UNC-10/RIM binds LAR intracellularly (for a review, see 56). However, neither nid-1(cg119) nor unc-10(md1117) enhanced $s d n-1(z h 20)$ or hse-5(tm472) (data not shown), indicating that while PTP-3/LAR influences D-type motor axon guidance this action is not going through standard LAR signaling.

The op462 mutation introduces a premature stop codon in zag-1, a gene coding for a $\mathrm{Zn}$ finger and homeodomain transcription factor that controls differentiation and axon guidance of multiple neuronal cell types, including the D-type 

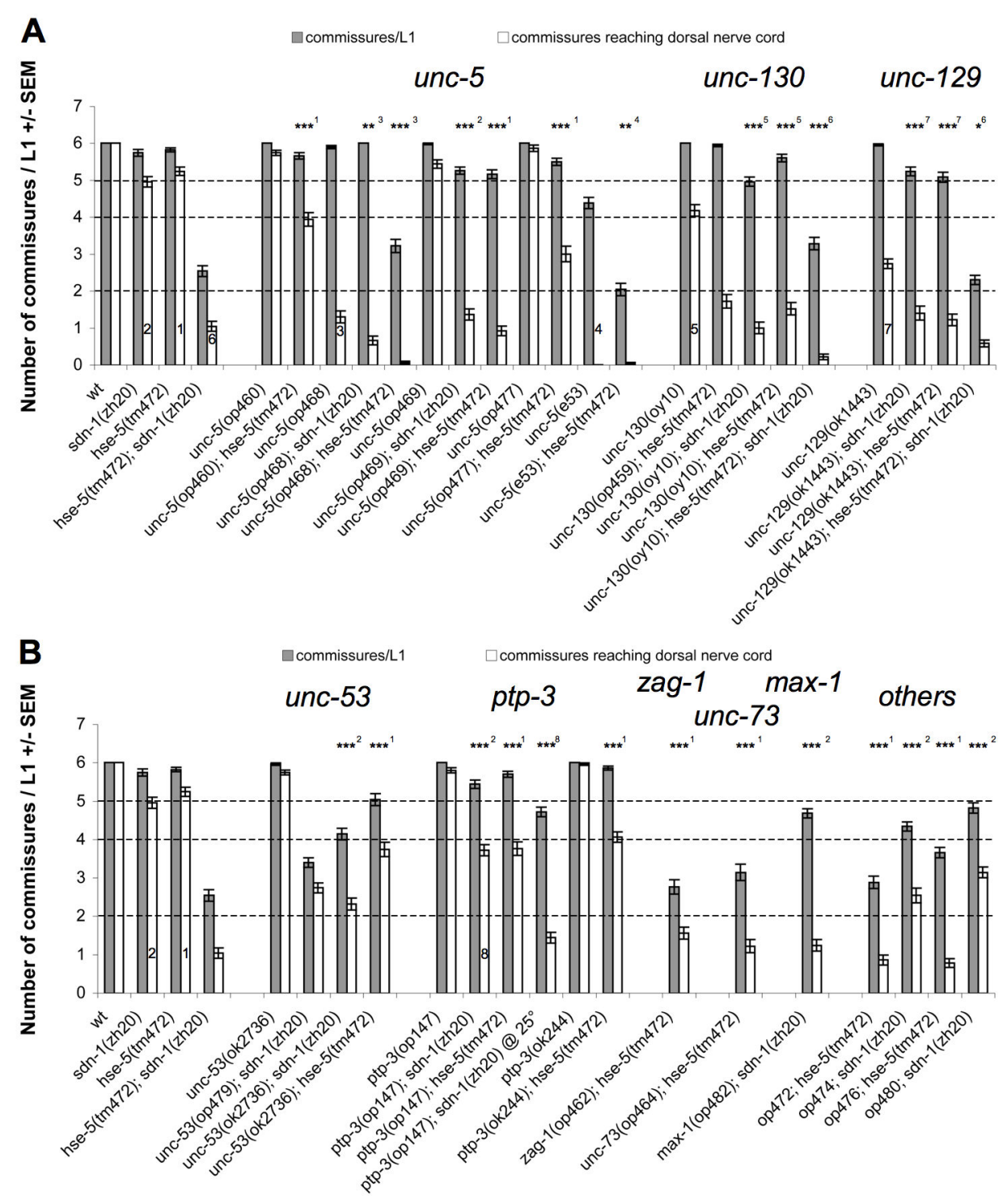

Figure 2. Axon guidance defects of sdn-1 and hse-5 enhancers. A: Netrin pathway mutants, as single mutants and in combination with $h s e-5(\operatorname{tm} 472)$ and $s d n-1(\mathrm{zh} 20)$. Of the unc-5 alleles only op 468 has a single mutant phenotype, but is still weaker than the e53 reference allele. Mutations in unc-129 and unc-130 show similar defects, both as single mutants as well as in double mutants with sdn-1(zh20) or hse-5(tm472). B: Mutations in unc-53 and ptp-3 enhance both sdn-1 and hse-5 mutations. op147 shows a strong temperature sensitive phenotype and is considered a strong allele at $25^{\circ}$. ok 244 on the other hand shows a weaker phenotype. We also isolated alleles in max-1, zag-1, and unc-73. op472 and op476 map to the same interval on chromosome I but whole genome sequencing failed to find a mutation in either strain. op474 and op 480 were difficult to map and therefore not characterized further. Grey bars represent the number of commissural axons growing away from the VNC; white bars indicate the number of commissural axons reaching the DNC. Dashed lines indicate limits according to Figure $1 \mathrm{~A}$. Numbers are from $50 \mathrm{~L} 1$ animals +/- SEM. Statistical test results are indicated as follows: ns $=$ not significant, ${ }^{*}=p<0.05,{ }^{* *}=p<0.005,{ }^{* * *}=p<0.0005$. Superscripts (also shown in the bars of the corresponding strains) indicate to which strain the comparison was made: 1: hse-5(tm472), 2: sdn-1(zh20), 3: unc-5(op468), 4: unc-5(e53), 5: unc-130(oy10), 6: hse-5(tm472); sdn-1(zh20), 7: unc-129(ok1443), 8: ptp-3(op147); sdn-1(zh20).

doi: 10.1371/journal.pone.0074908.g002

motor neurons, likely by regulating the expression of as yet unknown transcriptional target genes [57]. We also identified in op464 a mutation in an intron of unc-73, a guanine nucleotide exchange factor known to affect multiple axon guidance decisions [58] (Figure 2B). Whether this intronic mutation is the sequence change leading to the D-type axon guidance defects 
is unclear. Since it affects the 5th base in the intron it could potentially lead to a splicing defect.

Furthermore, we found a premature stop codon in the gene zfp-1 (Figure 3). ZFP-1 has not previously been described to act in UNC-6/Netrin signaling or HSPG related signaling. We therefore decided to focus our attention on this gene in order to understand how it influences HSPG dependent Netrin signaling.

\section{Axon guidance defects of zfp-1 and lin-35 mutants depend on the presence of the oxls12 transgene}

ZFP-1 is a zinc finger protein that has been found to control C. elegans vulval development by counteracting a large group of genes called synthetic multivulva (SynMuv) genes [36]. SynMuv genes can be separated into three classes, SynMuvA/B/C. While single mutants of any SynMuv gene show no aberrant vulva development, double mutants of two genes belonging to different SynMuv classes will result in the typical multivulval phenotype. ZFP-1 has also been suggested to act in the RNAi pathway, again counteracting SynMuv genes $[59,60]$. The human homolog of $z f p-1$ is called AF10 and is involved in pediatric acute myeloid leukemia (AML) [61] making zfp-1 an interesting gene to explore.

The op481 mutation introduces a premature stop codon in the ZFP-1 open reading frame (Table 1). A second zfp-1 allele, ok554, deletes part of the gene and shows an identical axon guidance phenotype as op481 (Figure 3A).

During our efforts to create an RNAi-sensitive strain containing $s d n-1(z h 20)$, we found that the double mutant lin-35(n745); sdn-1(zh20) displayed D-type axon guidance defects even without RNAi (Figure 3A). lin-35, a member of the SynMuv B class of genes, codes for the $C$. elegans homolog of the human Retinoblastoma tumor suppressor protein $(p R b)$ (for a comprehensive review of $\mathrm{pRb}$ function in cancer and development see 62). The diversity of functions of $p R b$ is a result of the ability of $p R b$ to interact with more than 150 different proteins and even act as an adaptor by interacting with more than one protein at a time [63].

Since zfp-1 is a SynMuv suppressor gene and lin-35 a SynMuv gene we wondered whether other SynMuv pathway genes would influence D-type axon guidance in a similar way. To test this we knocked down all the known SynMuv genes [64] and SynMuv suppressors [36] by RNAi. However, none of the genes tested resulted in a similar phenotype as zfp-1 or lin-35 (Table S1). In further attempts we explored the influence of Argonaute genes as well as genes involved in chromatin remodeling, but again failed to find any strong enhancers (Table S1).

The oxls12 D-type reporter transgene we used for our screen and all subsequent analyses contains multiple copies of the genes lin-15A and lin-15B as a co-injection marker. Both genes are part of the same operon known as the lin-15AB locus. Mutations affecting both genes together result in a strong SynMuv phenotype. Since lin-15AB are SynMuv genes themselves, we developed concerns regarding the use of a transgene such as ox/s12 that will lead to the overexpression of lin-15AB. To address this issue, we tested the phenotype of zfp-1(ok554); sdn-1(zh20) and zfp-1(ok554) hse-5(tm472) animals using the transgene ox/s268, which contains the same gfp reporter (unc-47::gfp) as ox/s12 but without any co-injection marker. Strikingly, in the oxls268 background, zfp-1(ok554) completely failed to enhance the defects of $s d n-1(z h 20)$ mutants and only weakly enhanced the axon guidance defects of hse-5(tm472) mutants (Figure 3A). Further experiments however suggested that lin-15AB overexpression in ox/s12 was not the cause for the enhancing effect of this transgene (data not shown).

Our findings prompted us to ask whether ox/s12 would generally influence D-type axon guidance and tested other mutations from our screen with different transgenes. Only one other gene, namely lin-35, showed an ox/s12 transgene dependence similar to $z f p-1$ (Figure 3A). It is important to note that none of the major genes involved in D-type axon guidance such as unc-5, unc-6, unc-40, unc-129, unc-130 as well as none of the genes found in our genetic screen or candidate gene approach showed axon guidance phenotypes that depended on the ox/s12 transgene (data not shown), except zfp-1 and lin-35. Taken together, our data clearly indicate that the axon guidance defects of zfp-1(ok554); sdn-1(zh20) and lin-35(n745); sdn-1(zh20) animals strongly depend on the presence of the oxls12 transgene and that the over-expression of lin-15AB from ox/s12 is most likely not relevant for this effect.

\section{Molecular characterization of oxls12}

Transgene-specific effects could occur due to genetic or epigenetic changes introduced by the transgene at its integration site. In order to test this possibility we sought to determine the precise site of ox/s12 integration. From previous genetic analysis, we expected ox/s12 to be less than 1cM away from $s d n-1$ on the $X$ chromosome (Figure 4A). Analysis of whole genome sequencing data of animals containing ox/s12 led to the identification of two locations with clear break points that are 232bp apart and represent a short deletion introduced by the integration of ox/s12, which is located in the 3' UTR of grd-1 and close to R08B4.5 (Figure 4B, C). Unfortunately we were unable to test the impact of $g r d-1$ or R08B4.5 in axon guidance with a mutation. However, RNAi against grd-1 and R08B4.5 failed to influence D-type motor axon guidance. Taken together, our observations support the conclusion that the transgene-specific effect of oxls12 is not due to disruption of a gene at the site of integration.

In order to gain insight into the size of ox/s12, we further analyzed the whole genome sequencing data (see Materials and Methods). This suggested that ox/s12 has a total size of about $3.6 \mathrm{Mb}$, increasing the size of the $X$ chromosome by approximately $20 \%$. Additionally, we performed qPCR for lin-15AB and unc-47 in ox/s12 animals and compared it to the N2 wild type. This approach led to a size estimation for ox/s12 of about $2.9 \mathrm{Mb}$. The fact that the qPCR data led to a lower estimation than the whole genome sequencing data can probably be explained by the fact that different parts of one construct are contained in different copy numbers.

Given the large size of oxls12 we hypothesized that the transgene dependence seen in zfp-1(ok554); sdn-1(zh20) animals could be a result of the increased size of the $X$ 

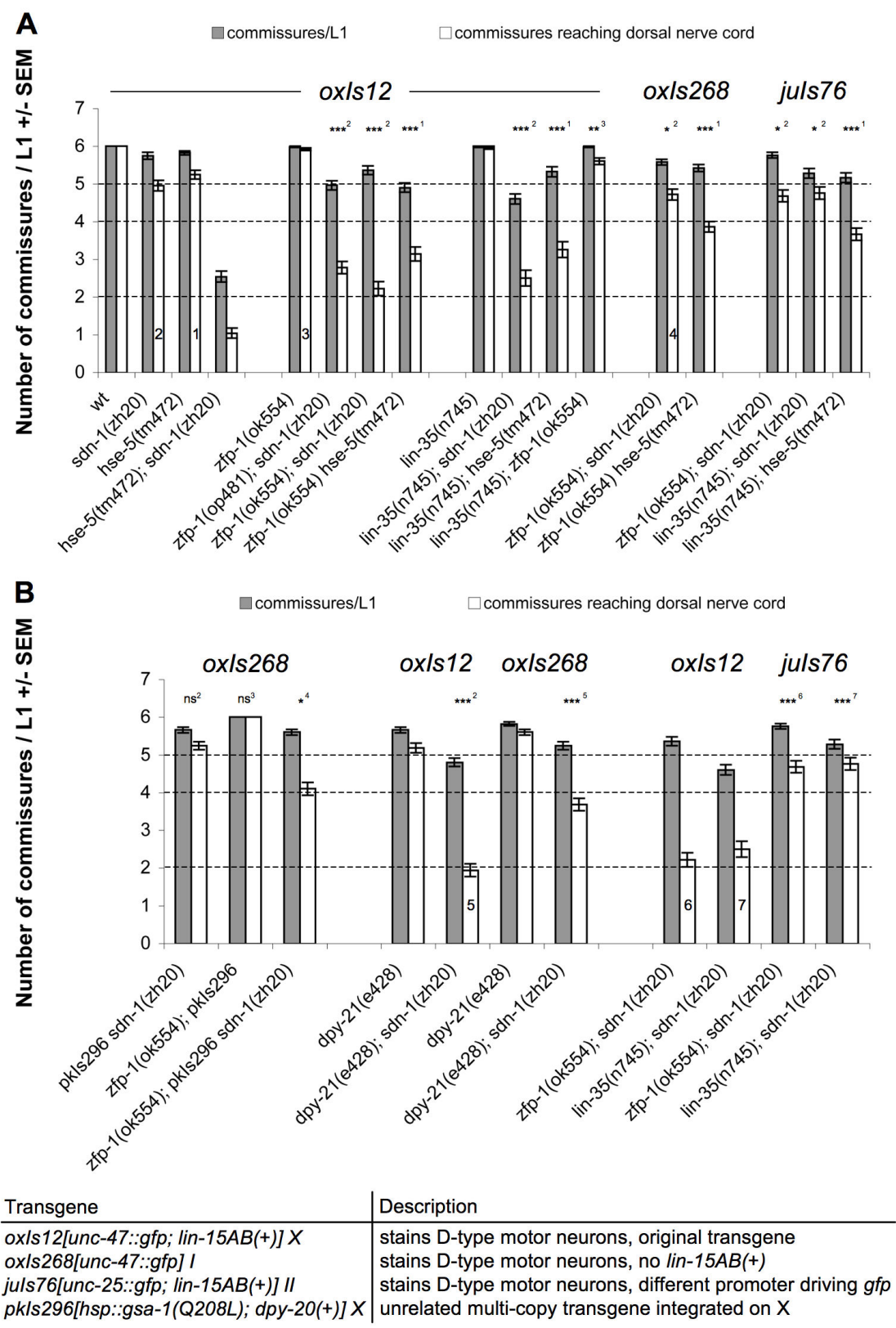

Figure 3. Mutations in zfp-1 or lin-35 enhance axon guidance defects in an oxls12-dependent manner. A: The $z f p-1$ (op481) point mutation, the zfp-1(ok554) deletion and lin-35(n745) lead to a very similar enhancement of defects when combined with sdn-1(zh20) or hse-5(tm472). However zfp-1(ok554) and lin-35(n745) are not enhancing each other. The defects of zfp-1(ok554); sdn-1(zh20) and lin-35(n745); sdn-1(zh20) disappear if oxls12 is replaced by either oxls268 or juls76. B: Attempts to reconstitute the defects. (i) pkls296 enlarges the X-chromosome of oxls268; zfp-1(ok554); sdn-1(zh20) animals leading to a slight increase in defects, however not to the level of $z f p-1$ (ok554); sdn-1(zh20) oxls12 animals (ii). The $d p y-21(\mathrm{e} 428)$ mutation behaves the same as zfp-1 or lin-35 mutations. The $d p y-21(\mathrm{e} 428)$; sdn-1(zh20) double mutant has severe D-type axon guidance defects if ox/s12 is present but not if oxls268 is used to stain the D-type motor neurons. The phenotypes of zfp-1(ok554); sdn-1(zh20) and lin-35(n745); sdn-1(zh20) with either oxls12 or juls76 are shown as comparison. Grey bars represent the number of commissural axons growing away from the VNC; white bars indicate the number of commissural axons reaching the DNC. Dashed lines indicate limits according to Figure 1A. Numbers are from 50 L1 animals +/- SEM. Statistical test results are indicated as follows: ns $=$ not significant, ${ }^{*}=$ $p<0.05,{ }^{* *}=p<0.005,{ }^{* * *}=p<0.0005$. Superscripts (also shown in the bars of the corresponding strains) indicate to which strain the comparison was made: 1: hse-5(tm472), 2: sdn-1(zh20), 3: zfp-1(ok554), 4: zfp-1(ok554); sdn-1(zh20); ox/s268, 5: dpy-21(e428); sdn-1(zh20) oxls12, 6: zfp-1(ok554); sdn-1(zh20) oxls12, 7: lin-35(n745); sdn-1(zh20) oxls12. The table summarizes the different transgenes used, indicates their composition and the chromosome in which they are integrated.

doi: 10.1371/journal.pone.0074908.g003 
A

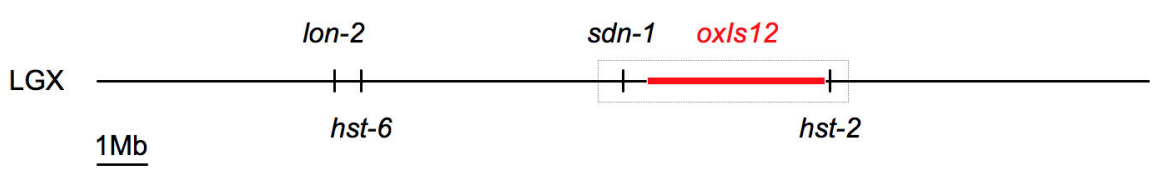

B

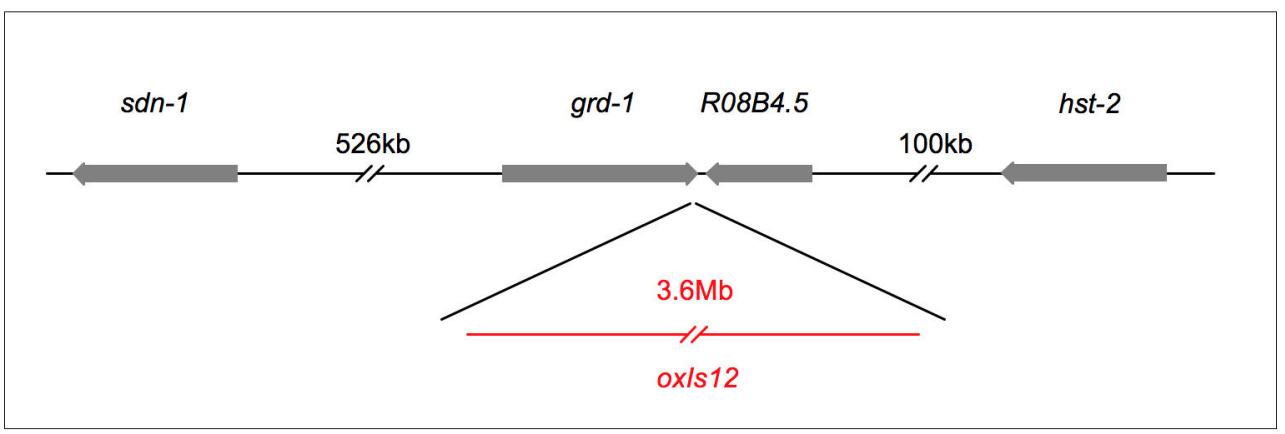

\section{C}

Left break point:

Reference sequence: AACACTATTTCATTTTCGTG TAGCTATTATGCGTCCTGTTTCCAGTAACATGATCAAATCGAGAAAAACCA... deleted in ox/s12:

grd-1 3'UTR:

Sequence in oxls12: AACACTATTTCATTTTCGTG ACAAATGCTACAGAGTTCTTGAAGTGGTGGCCTAACTACGGCTACACTAGA... vector sequence:

Right break point:

Reference sequence: ...GTCGCTACCAACAGCCGCAGTTCGCAATGTTCAAATTCATTAATTCCCGTT TTCAAGGAAAACTGGAATCA deleted in ox/s12:

Sequence in ox/s12: ...ATCTGCTGCTTGCAAACAAAAAAACCACCGCTACCAGCTTCAAGgAAAAC TTCAAGGAAAACTGGAATCA vector sequence:

Figure 4. Molecular characterization of oxIs12. A: X chromosome with the integrated transgene ox/s12, drawn to scale. ox/s12 is approximately $3.6 \mathrm{Mb}$ in size and thus substantially enlarges the $\mathrm{X}$ chromosome. B: Enlargement of the boxed region in A. Whole genome sequencing data place oxls12 within the 3' UTR region of grd-1, close to R08B4.5. C: Break points introduced by oxls12. The insertion introduced a deletion of 232bp (blue boxes) including the last 25bp of the 3' UTR of grd-1 (grey arrow) and a large part of the intergenic region between grd-1 and R08B4.5. The sequencing reads spanning the break points also reveal a small part of the transgene sequence (red boxes). In both cases a BLAST search indicates the presence of vector backbone sequence, which would usually not be found in the $C$. elegans genome.

doi: 10.1371/journal.pone.0074908.g004

chromosome due to the integration of the multi-copy transgene oxls12 on X. We therefore crossed pkls296[gsa-1(Q208L); $d p y-20(+)]$, another unrelated multi-copy transgene integrated on X, into oxls268; zfp-1(ok554); sdn-1(zh20) animals (oxIs268 is integrated on chromosome I). qPCR for the components of pkls296 indicated that $p k l s 296$ is probably slightly smaller in size compared to oxls12. Indeed, oxls268; zfp-1(ok554); sdn-1(zh20) pkls296 animals showed increased defects compared to oxls268; zfp-1(ok554); sdn-1(zh20), although not to the level of defects seen in zfp-1(ok554); sdn-1(zh20) oxls12 (Figure 3B).

Increasing the size of the $X$ chromosome could interfere with dosage compensation. If the dosage compensation complex (DCC) has to cover a bigger chromosome, this could lead to a reduction of DCC density and consequently to an increased transcription of X-linked genes. Indeed, many of the genes with a link to D-type motor axon guidance and HSPGs are located on the $X$ chromosome, e.g. unc- 6 , lon-2, sdn-1, hst-2 and hst-6. If loss of zfp-1 or lin-35 function were to reduce dosage compensation efficiency, then a mutation in the gene $d p y-21$, a member of the DCC [38], might lead to similar axon guidance defects in our specific setup. Indeed, the $d p y-21(e 428)$; $s d n-1(z h 20)$ oxls12 strain had increased D-type motor axon guidance defects compared to the oxIs268; $d p y-21(e 428)$; $s d n-1(z h 20)$ strain (Figure 3B). This finding clearly shows that the integration of a multi-copy transgene on the $X$ chromosome in the sensitive genotype used in our mutagenesis screen to find novel HSPG related axon guidance genes has led to the 
discovery of two genes that influence axon guidance in an indirect way.

\section{Discussion}

\section{The HSPG network of D-type motor axon guidance}

Our efforts to shed light on the role played by HSPGs and HS modifying enzymes during guidance of D-type motor axons clearly showed that SDN-1/Syndecan and LON-2/Glypican have parallel functions in this process. Other core proteins such as GPN-1/Glypican, CLE-1/Collagen XVII, UNC-52/ Perlecan or AGR-1/Agrin did not seem to be involved. Minor defects could however be observed, which might be the result of pleiotropic defects, since it is very likely that each core protein has a wide range of functions.

Our analysis of the modifying enzymes revealed that mutations in hst-2, hse-5, and hst-6 clearly enhance $s d n-1(z h 20)$ but not lon-2(e678), indicating that they all modify HS chains of LON-2, and that they either do not modify SDN-1, or that these modifications are not important for SDN-1 function in D-type axon guidance. HS-3-O sulfotransferase activity coming from hst-3.1 or hst-3.2 does not seem to be important for D-type motor axon guidance, at least under the conditions and in the genotypes tested. Surprisingly, we found that hst-6 hst-2 and hse-5; hst-6 double mutants, but not hse-5; hst-2, showed enhanced D-type motor axon guidance defects. Since the lon-2 hst-6 sdn-1 triple mutant was indistinguishable from the lon-2 sdn-1 double mutant, we hypothesized that HST-6 is indeed acting on the HS chains of both LON-2 and SDN-1, but that the lack of modification on SDN-1 is not strongly detrimental.

The strain with the strongest defects is the hse-5(tm472); $s d n-1(z h 20)$ double mutant. Theoretically this phenotype should be phenocopied by mutating all the relevant core proteins. However the double mutant of lon-2 and sdn-1, which were shown to act in D-type axon guidance, has less dramatic defects. This could point to the presence of a third core protein or it could be the result of a dominant negative function of HS chains on LON-2, which lack the modification introduced by HSE-5 and therefore have altered binding affinities. The second explanation could be tested in an hse-5(tm472); lon-2(e678) sdn-1(zh20) triple mutant, which should then be phenotypically identical to the lon-2(e678) sdn-1(zh20) double mutant. Unfortunately, this triple mutant is lethal and lon-2 RNAi in the sdn-1(zh20) oxls12 and hse-5(tm472); sdn-1(zh20) oxls12 background had no effect on axon guidance (data not shown).

Taken together, the data indicate that there is a tight network of HSPGs and HS modifying enzymes influencing dorsal guidance of D-type motor axons. The ready availability of mutations in all HSPG core proteins and HS modifying enzymes enabled us to describe the HSPG network relevant for efficient dorsal axon guidance in the worm and establish the HS code for both involved core proteins. The HS chains of LON-2 need to be modified by HST-2, HSE-5 and HST- 6 to be able to exert their function. HS chains on SDN-1 seem to require HST-6 function only.

\section{A screen for genes acting upstream or downstream of HSPGs during D-type motor axon guidance}

In order to find new genes involved in HSPG-dependent axon guidance, we designed two approaches for a forward genetic screen. We chose to use either $s d n-1(z h 20)$ or hse-5(tm472) as sensitive backgrounds, because the double mutant of these two genes showed the most severe axon guidance defects in D-type motor axons and we assumed that the function of those two genes would be most divergent. Furthermore, using a sensitive mutant background should allow us to recover mutations that only have a weak phenotype on their own, similar to the two background mutations. For both backgrounds we screened about 11'000 haploid genomes. It became clear upon mapping that we did not saturate the genome with the mutagenesis because (i) we failed to recover the positive controls sdn-1 and hse-5 or some obvious candidates such as unc- 6 and unc- 40 and (ii) all the genes found to be mutated in a candidate were only recovered once, with the clear exception of unc- 5 for which we found 4 different alleles. It is certainly remarkable that unc-5 is so prone to the accumulation of mutations in our screening set up. Interestingly, the three point mutants op460, op469 and op 477 alone have little or no effect on axon guidance; a phenotype can be observed only when combined with a mutation in sdn-1 or hse-5, i.e. when HSPG related signaling is impaired. There are multiple ways of interpreting this finding: a) HSPGs could bind via their HS chains to UNC-5 to facilitate signaling through it or locally concentrate a ligand, which may make the amino acid sequence of UNC-5 particularly sensitive to changes because changing the amino acid sequence could alter the HS binding efficiency. b) HSPGs could influence a signaling pathway acting in parallel to UNC-6/Netrin, such as the Slit/ Robo pathway that has been shown to act in ventral axon guidance $[65,66]$. Altering signaling through this parallel pathway could render D-type motor axons particularly sensitive to any further changes in axon guidance signaling. It is interesting to note that the double mutant hse-5(tm472); unc-5(e53) and the two triple mutants hse-5(tm472); unc-129(ok1443); sdn-1(zh20) and unc-130(oy10); hse-5(tm472); sdn-1(zh20) all resemble hse-5(tm472); $s d n-1(z h 20)$ double mutants, hinting that UNC-5, HSE-5, SDN-1 and UNC-129/130 could possibly function in a common pathway.

The initial aim of the screen was to find genes that enhance e.g. $s d n-1(z h 20)$ and fail to enhance $h s e-5(t m 472)$. Remarkably, none of the enhancers isolated in the screen were found to have this effect. Rather, all mutations isolated enhanced both sdn-1(zh20) and hse-5(tm472) to similar levels. This indicates that the function of SDN-1 and LON-2 could converge on a common pathway during guidance of D-type motor axons. The results from the screen suggest that this common pathway could be UNC-6/Netrin signaling (Figure 5). The sensitivity for mutations in unc- 5 seen in our screen could suggest that HS chains attached to LON-2 and/or SDN-1 may bind to UNC-5. Alternatively, LON-2 and/or SDN-1 could bind via their HS chains to the UNC-5 ligands UNC-6/Netrin or UNC-129/TGF $\beta$, either to increase their local concentration or to facilitate their interaction with UNC-5. Additionally a third 
pathway in parallel to UNC-6/Netrin signaling could be influenced by HSPGs. Since LON-2 and SDN-1 were shown to carry distinct HS codes and are expressed in different tissues (hypodermis and neurons, respectively) they may interact differentially with UNC-6, UNC-129 or other unknown ligands. Finally, SDN-1 could also act as a receptor in its own right and activate a signal transduction cascade upon ligand binding.

The finding that the transmembrane tyrosine phosphatase PTP-3 plays a role in D-type motor axon guidance suggests a requirement for tyrosine phosphorylation in this process. Drosophila LAR, the homolog of PTP-3, has been shown to bind to HS chains on Syndecan and Glypican [54,55]. A similar function for PTP-3 is therefore likely. Furthermore, we describe for the first time a function in dorsal axon guidance for UNC-53, a gene involved in cytoskeleton remodeling previously shown to participate in longitudinal axon guidance (Figure 5). Whether PTP-3 and UNC-53 influence Netrin signaling or act in parallel pathways remains an open question.

\section{The large multi-copy transgene oxls12 influences $D$ - type axon guidance in combination with a mutation in zfp-1 or lin-35}

In our search for novel components that influence HSPG dependent $\mathrm{D}$-type motor axon guidance, we also identified two apparently unrelated genes affecting the process, zfp-1 and lin-35. zfp-1 has been shown to counteract SynMuv genes [36] and to have a positive influence on the RNAi pathway $[59,60]$. On the other hand, many SynMuv genes have a negative influence on RNAi [67], which is why lin-35 mutants are often used as sensitive background for RNAi experiments. However, no other SynMuv or SynMuv suppressor genes showed the same phenotypes as lin-35(n745) or zfp-1(ok554), suggesting that their influence on axon guidance is not the representation of a general effect of these two classes of genes.

During our investigation of the general effect on axonal guidance of SynMuv genes, we noted that the oxls12[unc-47::gfp; lin-15AB(+)] transgene used to visualize the D-type motor neurons was required to induce D-type motor axon guidance defects in zfp-1(ok554); sdn-1(zh20) and lin-35(n745); sdn-1(zh20) animals. Two other transgenes (ox/s268[unc-47::gfp] and juls76[unc-25::gfp; lin-15AB(+)]) did not provoke major D-type axon guidance defects in those two double mutants, suggesting that neither the gfp construct nor the marker used in the transgenes are playing a role. ox/s12 is not generally influencing D-type motor axon guidance. The transgene dependence phenotype is very specific for $z f p-1$ and lin-35 mutations in combination with a second mutation in an HSPG network gene.

\section{Multi-copy transgenes integrated on $X$ could interfere with dosage compensation}

Our analysis of ox/s12 resulted in an estimated size of approximately $3.6 \mathrm{Mb}$. This is a significant amount of additional DNA in an animal with a wild-type genome size of about $100 \mathrm{Mb}$ and is increasing the size of the $X$ chromosome, which is $17.7 \mathrm{Mb}$ long, by approximately $20 \%$. Since many genes contained in the HSPG network (Figure 1) (hst-6, lon-2, sdn-1, hst-2) as well as unc-6/Netrin are located on X (Figure 5) we

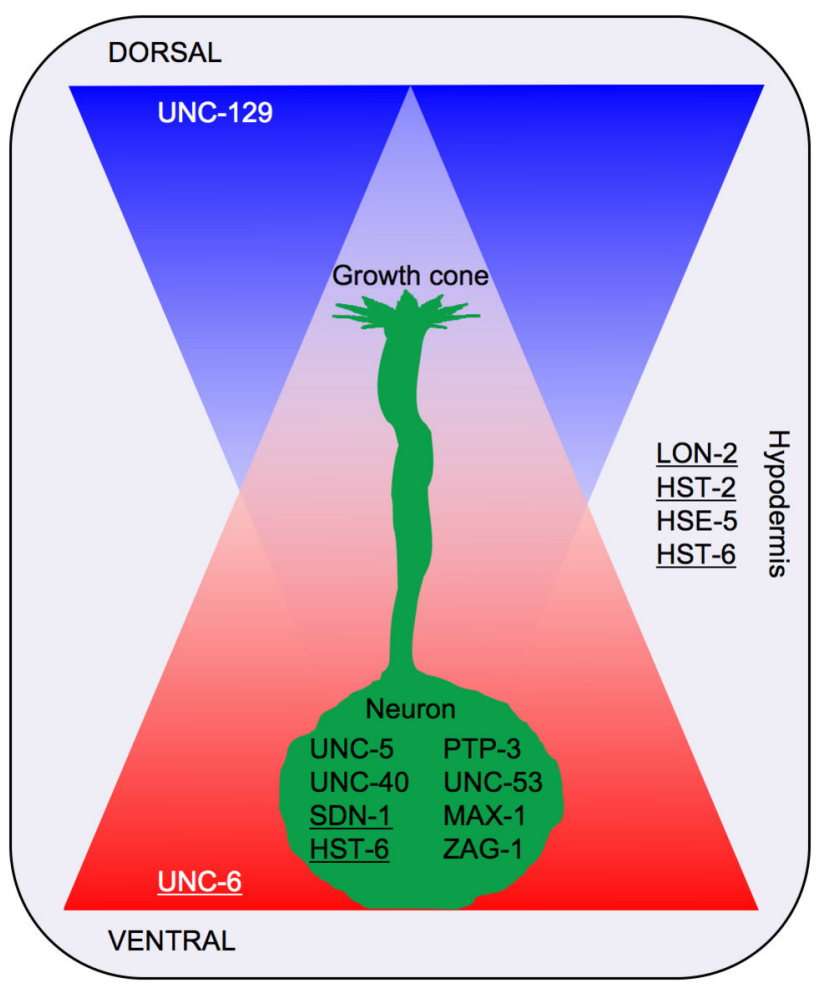

Figure 5. Factors involved in dorsal axon guidance of Dtype motor neurons. The two UNC-5 ligands UNC-6 (red) and UNC-129 (blue) form opposing gradients with high concentrations of UNC-6 ventrally and high UNC-129 concentrations on the dorsal side of the worm. The ventrally outgrowing growth cone (green) initially uses the UNC-5 receptor to respond to high UNC-6 concentrations. The more the growth cone approaches the dorsal side, the lower the UNC-6 concentration becomes while the concentration of UNC-129 increases, which is thought to induce a switch in UNC-6 signaling in the growth cone to UNC-5 + UNC-40 signaling. This enables the growth cone to maintain its response to the repulsive UNC-6 signal [24]. The HSPG SDN-1 is expressed in the neuron [5] and requires HST-6 function to modify its HS chains. SDN-1 could, through its HS chains, influence the interaction of UNC-6 and/or UNC-129 with UNC-5. Our genetic data suggest that PTP-3 is playing a role in this process too, but it remains an open question what its precise function is. Furthermore UNC-53 is involved in cytoskeletal remodeling [52] and MAX-1 [34] and ZAG-1 [57] are transcription factors with a possible function in the neuron. The HSPG LON-2 is expressed in the hypodermis [50]. Its function could be to establish and/or maintain the gradients of UNC-6 and/or UNC-129 to provide an ideal substrate for the growth cone on its way to the dorsal side. For this function LON-2 requires the enzymes HST-2, HSE-5 and HST-6 to modify its HS chains. Underlined protein names indicate that these genes are located on the $\mathrm{X}$ chromosome.

doi: 10.1371/journal.pone.0074908.g005 
hypothesized that the change in size of the $X$ chromosome is what made ox/s12 special.

ox/s12 could disrupt dosage compensation by diluting the dosage compensation complex (DCC) across the whole enlarged $X$ chromosome. This would lead to an upregulation of genes on the $X$ chromosome and ultimately to D-type axon guidance defects when combined with an sdn-1(zh20) mutation, since there are many other genes on $X$ that are relevant for proper guidance of D-type motor axons. A clear confirmation of this hypothesis came from the finding that the dosage compensation mutant $d p y-21(e 428)$ showed the same phenotype as zfp-1 and lin-35 mutants (Figure 3B). In dpy-21 loss of function mutants the $X$ chromosome is transcriptionally upregulated [68]. However, a microarray study in zfp-1(ok554) and lin-35(n745) mutants did not detect an accumulation of $\mathrm{X}$ linked genes among the genes upregulated in these two mutant backgrounds. Furthermore, none of the core DCC components were significantly up- or downregulated in zfp-1(ok445) or lin-35(n745) mutants [69]. This clearly indicates that ZFP-1 and LIN-35 are not playing a direct role in dosage compensation per se. Nevertheless, it is certainly remarkable that in combination with oxls12, mutations in zfp-1, lin-35 and dpy-21 behave similarly.

Taken together, our data show that the use of large multicopy transgenes in $C$. elegans can be problematic, at least in combination with mutations in certain genes. Genetic screens are nowadays usually performed in sensitized backgrounds, which clearly increase the risk of finding candidates whose genetic link to the process under study is difficult to establish. In our case we were screening for axon guidance genes. However, our in-depth analysis of zfp-1 and lin-35 proved that they are clearly not "axon guidance" genes. Rather, loss of zfp-1 or lin-35 likely disrupts the normal gene expression pattern, indirectly leading to axon guidance defects. Which genes mediate this effect remains to be determined. The phenocopy of the zfp-1 phenotype by the DCC mutant $d p y-21$ suggests that at least one critical target gene is on the $X$ chromosome.

A number of interesting questions remain open regarding the genes involved in the HSPG network and their function in dorsal axon guidance. Which signaling pathway is influenced by HSPGs? Is it Netrin signaling, the major dorsal guidance system or a parallel pathway such as Slit/Robo? What are the distinct functions and interaction partners of the two core proteins SDN-1 and LON-2? What is the role played by PTP-3 during dorsal axon guidance? What is the function of UNC-53 in cytoskeleton remodeling during dorsal axon guidance? Further biochemical experiments might provide answers to these questions.

\section{Supporting Information}

Figure S1. Estimation of the size of oxls12. Coverage analysis of the lin-15AB locus, which was used as co-injection marker to create the extrachromosomal array used for generation of oxls12. Sequencing reads originating from the lin-15AB fragments in oxls12 will align at the endogenous lin-15AB locus and therefore lead to an increase of coverage in that area. While the average genome coverage was $70.35 \mathrm{x}$, it increased to $11030 \mathrm{x}$ across the lin-15AB locus (indicated by red line). The part of the lin-15AB locus contained in the transgene can be determined precisely since coverage at the beginning and the end jumps from around $50 x$ to over $4000 x$, and increases further from base to base (see enlargements of beginning and end).

(TIF)

Figure S2. Effect of HSPG core protein and HS modifying enzyme mutants on D-type motor axon guidance. Strains are ordered according to the severity of the defects. The first class contains all the single mutants as well as all the double mutants that show no/weak defects. The groups "weak enhancers" and "strong enhancers" are mostly the same strains as shown in Figure 1, additionally the strains containing unc-52(e444) are shown. Note that while unc-52(e444) weakly enhances $s d n-1(z h 20)$ it does not enhance the lon-2(e678) $s d n-1(z h 20)$ double mutant and is therefore considered not to play a role in D-type motor axon guidance. Grey bars represent the number of commissural axons growing away from the ventral nerve cord (VNC); white bars indicate the number of commissural axons reaching the DNC. Dashed lines indicate limits according to Figure $1 \mathrm{~A}$. Numbers are from 50 L 1 animals $+/$ - SEM. For statistical evaluation of relevant strains see Figure $1 \mathrm{~A}$.

(TIF)

Figure S3. Outline of the screen. Young adult P0 animals carrying either $h s e-5(t m 472)$ or $s d n-1(z h 20)$ as a background mutation and the ox/s12 transgene to label D-type motor neurons were mutagenized with EMS. Three P0 animals were put on one $9 \mathrm{~cm}$ plate and transferred to fresh plates twice a day. F1 animals were allowed to lay eggs for about $12 \mathrm{~h}$ before being removed from the plate. F2 animals were screened under a dissection fluorescence microscope for candidates phenocopying the strong D-type axon guidance defects of hse-5(tm472); sdn-1(zh20) double mutants. Candidates were singled out and their progeny rescreened a couple of days later to confirm the phenotype.

(TIF)

Table S1. Summary of all genes treated with RNAi. (DOC)

\section{Acknowledgements}

We thank the Caenorhabditis Genome Center (funded by NIH Office of Research Infrastructure Programs, p40 OD010440) and various $C$. elegans researchers for providing strains, the international $C$. elegans Gene Knockout Consortium and the National Bioresource Project in Japan for deletion alleles, the group of Dr. Christian Beisel at the ETH in Basel for Illumina sequencing and Dr. Gabriele Cozzi for help with statistical analysis. 


\section{Author Contributions}

Conceived and designed the experiments: SG CR $\mathrm{MOH}$. Performed the experiments: SG. Analyzed the data: SG.

\section{References}

1. Tessier-Lavigne M, Goodman CS (1996) The molecular biology of axon guidance. Science 274: 1123-1133. doi:10.1126/science. 274.5290.1123. PubMed: 8895455.

2. Irie A, Yates EA, Turnbull JE, Holt CE (2002) Specific heparan sulfate structures involved in retinal axon targeting. Development 129: 61-70. PubMed: 11782401.

3. Johnson KG Ghose A, Epstein E, Lincecum J, O'Connor MB et al. (2004) Axonal heparan sulfate proteoglycans regulate the distribution and efficiency of the repellent slit during midline axon guidance. Curr Biol 14: 499-504. doi:10.1016/j.cub.2004.02.005. PubMed: 15043815.

4. Steigemann P, Molitor A, Fellert S, Jäckle H, Vorbrüggen G (2004) Heparan sulfate proteoglycan syndecan promotes axonal and myotube guidance by slit/robo signaling. Curr Biol 14: 225-230. doi:10.1016/ j.sbi.2004.03.002. PubMed: 14761655.

5. Rhiner C, Gysi S, Fröhli E, Hengartner MO, Hajnal A (2005) Syndecan regulates cell migration and axon guidance in $C$. elegans. Development 132: 4621-4633. doi:10.1242/dev.02042. PubMed: 16176946.

6. Smart AD, Course MM, Rawson J, Selleck S, Van Vactor D et al. (2011) Heparan sulfate proteoglycan specificity during axon pathway formation in the Drosophila embryo. Dev Neurobiol 71: 608-618. doi: 10.1002/dneu.20854. PubMed: 21500363

7. Bernfield M, Götte M, Park PW, Reizes O, Fitzgerald ML et al. (1999) Functions of cell surface heparan sulfate proteoglycans. Annu Rev Biochem 68: 729-777. doi:10.1146/annurev.biochem.68.1.729. PubMed: 10872465.

8. Yamaguchi $Y$ (2001) Heparan sulfate proteoglycans in the nervous system: their diverse roles in neurogenesis, axon guidance, and synaptogenesis. Semin Cell Dev Biol 12: 99-106. doi:10.1006/scdb. 2000.0238. PubMed: 11292375.

9. Rhiner C, Hengartner MO (2006) Sugar antennae for guidance signals: syndecans and glypicans integrate directional cues for navigating neurons. ScientificWorldJournal 6: 1024-1036. doi:10.1100/tsw. 2006.202. PubMed: 16937008

10. Van Vactor D, Wall DP, Johnson KG (2006) Heparan sulfate proteoglycans and the emergence of neuronal connectivity. Curr Opin Neurobiol 16: 40-51. doi:10.1016/j.conb.2006.01.011. PubMed: 16417999.

11. Mizuguchi S, Dejima K, Nomura K (2009) Sulfation and related genes in Caenorhabditis elegans. Trends Glycosci Glycotechnol 21: 179-191. doi:10.4052/tigg.21.179.

12. Esko JD, Selleck SB (2002) Order out of chaos: assembly of ligand binding sites in heparan sulfate. Annu Rev Biochem 71: 435-471. doi: 10.1146/annurev.biochem.71.110601.135458. PubMed: 12045103.

13. Bülow HE, Hobert $O$ (2006) The molecular diversity of glycosaminoglycans shapes animal development. Annu Rev Cell Dev Biol 22: 375-407. doi:10.1146/annurev.cellbio.22.010605.093433. PubMed: 16805665.

14. Lin X (2004) Functions of heparan sulfate proteoglycans in cell signaling during development. Development 131: 6009-6021. doi: 10.1242/dev.01522. PubMed: 15563523

15. Lee JS, Chien CB (2004) When sugars guide axons: insights from heparan sulphate proteoglycan mutants. Nat Rev Genet 5: 923-935. doi:10.1038/nrg1490. PubMed: 15573124

16. Bülow HE, Hobert $O$ (2004) Differential sulfations and epimerization define heparan sulfate specificity in nervous system development. Neuron 41: 723-736. doi:10.1016/S0896-6273(04)00084-4. PubMed: 15003172 .

17. Bülow HE, Tjoe N, Townley RA, Didiano D, van Kuppevelt TH et al. (2008) Extracellular sugar modifications provide instructive and cellspecific information for axon-guidance choices. Curr Biol 18: 1978-1985. doi:10.1016/j.cub.2008.11.023. PubMed: 19062279.

18. Holt CE, Dickson BJ (2005) Sugar codes for axons? Neuron 46: 169-172. doi:10.1016/j.neuron.2005.03.021. PubMed: 15848796.

19. Hedgecock EM, Culotti JG, Hall DH (1990) The unc-5, unc-6, and unc-40 genes guide circumferential migrations of pioneer axons and mesodermal cells on the epidermis in C. elegans. Neuron 4: 61-85. doi: 10.1016/0896-6273(90)90444-K. PubMed: 2310575

20. Chisholm A, Tessier-Lavigne M (1999) Conservation and divergence of axon guidance mechanisms. Curr Opin Neurobiol 9: 603-615. doi: 10.1016/S0959-4388(99)00021-5. PubMed: 10508749.
Contributed reagents/materials/analysis tools: SF. Wrote the manuscript: SG. Helped with preparation of the manuscript: CR MOH SF DGM.

21. Huber AB, Kolodkin AL, Ginty DD, Cloutier JF (2003) Signaling at the growth cone: ligand-receptor complexes and the control of axon growth and guidance. Annu Rev Neurosci 26: 509-563. doi:10.1146/ annurev.neuro.26.010302.081139. PubMed: 12677003.

22. Lim YS, Mallapur S, Kao G, Ren XC, Wadsworth WG (1999) Netrin UNC-6 and the regulation of branching and extension of motoneuron axons from the ventral nerve cord of Caenorhabditis elegans. J Neurosci 19: 7048-7056. PubMed: 10436059.

23. Durbin RM (1987). PhD thesis

24. MacNeil LT, Hardy WR, Pawson T, Wrana JL, Culotti JG (2009) UNC-129 regulates the balance between UNC-40 dependent and independent UNC-5 signaling pathways. Nat Neurosci 12: 150-155. doi: 10.1038/nn.2256. PubMed: 19169249.

25. Belenkaya TY, Han C, Yan D, Opoka RJ, Khodoun M et al. (2004) Drosophila Dpp morphogen movement is independent of dynaminmediated endocytosis but regulated by the glypican members of heparan sulfate proteoglycans. Cell 119: 231-244. doi:10.1016/j.cell. 2004.09.031. PubMed: 15479640.

26. Matsumoto $Y$, Irie $F$, Inatani $M$, Tessier-Lavigne $M$, Yamaguchi $Y$ (2007) Netrin-1/DCC signaling in commissural axon guidance requires cell-autonomous expression of heparan sulfate. J Neurosci 27: 4342-4350. doi:10.1523/JNEUROSCI.0700-07.2007. PubMed: 17442818.

27. Brenner S (1974) The genetics of Caenorhabditis elegans. Genetics 77: 71-94. PubMed: 4366476

28. Lu X, Horvitz HR (1998) lin-35 and lin-53, two genes that antagonize a C. elegans Ras pathway, encode proteins similar to $\mathrm{Rb}$ and its binding protein RbAp48. Cell 95: 981-991. doi:10.1016/ S0092-8674(00)81722-5. PubMed: 9875852.

29. Hammarlund M, Nix P, Hauth L, Jorgensen EM, Bastiani M (2009) Axon regeneration requires a conserved MAP kinase pathway. Science 323: 802-806. doi:10.1126/science.1165527. PubMed: 19164707.

30. Ackley BD, Crew JR, Elamaa H, Pihlajaniemi T, Kuo CJ et al. (2001) The NC1/endostatin domain of Caenorhabditis elegans type XVIII collagen affects cell migration and axon guidance. J Cell Biol 152: 1219-1232. doi:10.1083/jcb.152.6.1219. PubMed: 11257122.

31. Harrington RJ, Gutch MJ, Hengartner MO, Tonks NK, Chisholm AD (2002) The C. elegans LAR-like receptor tyrosine phosphatase PTP-3 and the VAB-1 Eph receptor tyrosine kinase have partly redundant functions in morphogenesis. Development 129: 2141-2153. PubMed: 11959824.

32. Rogalski TM, Gilchrist EJ, Mullen GP, Moerman DG (1995) Mutations in the unc-52 gene responsible for body wall muscle defects in adult Caenorhabditis elegans are located in alternatively spliced exons. Genetics 139: 159-169. PubMed: 7535716.

33. Sarafi-Reinach TR, Sengupta $P(2000)$ The forkhead domain gene unc-130 generates chemosensory neuron diversity in C. elegans. Genes Dev 14: 2472-2485. doi:10.1101/gad.832300. PubMed: 11018015.

34. Huang $X$, Cheng $H J$, Tessier-Lavigne $M$, Jin $Y$ (2002) MAX-1, a novel $\mathrm{PH} /$ MyTH4/FERM domain cytoplasmic protein implicated in netrinmediated axon repulsion. Neuron 34: 563-576. doi:10.1016/ S0896-6273(02)00672-4. PubMed: 12062040.

35. Tecle E, Diaz-Balzac CA, Bülow HE (2013) Distinct 3-O-sulfated heparan sulfate modification patterns are required for kal-1-dependent neurite branching in a context-dependent manner in Caenorhabditis elegans. G3 Bethesda 3: 541-52.

36. Cui M, Kim EB, Han M (2006) Diverse chromatin remodeling genes antagonize the $\mathrm{Rb}$-involved SynMuv pathways in $C$. elegans. PLOS Genet 2: e74. doi:10.1371/journal.pgen.0020074. PubMed: 16710447.

37. Kennedy S, Wang D, Ruvkun G (2004) A conserved siRNA-degrading RNase negatively regulates RNA interference in $C$. elegans. Nature 427: 645-649. doi:10.1038/nature02302. PubMed: 14961122.

38. Yonker SA, Meyer BJ (2003) Recruitment of C. elegans dosage compensation proteins for gene-specific versus chromosome-wide repression. Development 130: 6519-6532. doi:10.1242/dev.00886. PubMed: 14660541.

39. Hudson ML, Kinnunen T, Cinar HN, Chisholm AD (2006) C. elegans Kallmann syndrome protein $\mathrm{KAL}-1$ interacts with Syndecan and 
Glypican to regulate neuronal cell migrations. Dev Biol 294: 352-365. doi:10.1016/j.ydbio.2006.02.036. PubMed: 16677626.

40. Mclntire SL, Reimer RJ, Schuske K, Edwards RH, Jorgensen EM (1997) Identification and characterization of the vesicular GABA transporter. Nature 389: 870-876. doi:10.1038/39908. PubMed: 9349821.

41. Korswagen HC, Park JH, Ohshima Y, Plasterk RH (1997) An activating mutation in a Caenorhabditis elegans Gs protein induces neural degeneration. Genes Dev 11: 1493-1503. doi:10.1101/gad.11.12.1493. PubMed: 9203577

42. Zipperlen P, Nairz K, Rimann I, Basler K, Hafen E et al. (2005) A universal method for automated gene mapping. Genome Biol 6: R19. doi:10.1186/gb-2005-6-2-r19. PubMed: 15693948

43. Maydan JS, Flibotte S, Edgley ML, Lau J, Selzer RR et al. (2007) Efficient high-resolution deletion discovery in Caenorhabditis elegans by array comparative genomic hybridization. Genome Res 17: 337-347. doi:10.1101/gr.5690307. PubMed: 17267812.

44. Flibotte S, Edgley ML, Maydan J, Taylor J, Zapf R et al. (2009) Rapid high resolution single nucleotide polymorphism-comparative genome hybridization mapping in Caenorhabditis elegans. Genetics 181: 33-37. PubMed: 18957702.

45. Sarin S, Prabhu S, O'Meara MM, Pe'er I, Hobert O (2008) Caenorhabditis elegans mutant allele identification by whole-genome sequencing. Nat Methods 5: 865-867. doi:10.1038/nmeth.1249. PubMed: 18677319.

46. Bigelow H, Doitsidou M, Sarin S, Hobert O (2009) MAQGene: software to facilitate $C$. elegans mutant genome sequence analysis. Nat Methods 6: 549. doi:10.1038/nmeth.f.260. PubMed: 19620971.

47. R: A language and environment for Statistical Computing, Vienna, Austria. Available: http://www.R-project.org.

48. Ackley BD, Kang SH, Crew JR, Suh C, Jin Y et al. (2003) The basement membrane components nidogen and type XVIII collagen regulate organization of neuromuscular junctions in Caenorhabditis elegans. J Neurosci 23: 3577-3587. PubMed: 12736328.

49. Merz DC, Alves G, Kawano T, Zheng H, Culotti JG (2003) UNC-52/ perlecan affects gonadal leader cell migrations in $C$. elegans hermaphrodites through alterations in growth factor signaling. Dev Biol 256: 173-186. PubMed: 12654300.

50. Gumienny TL, MacNeil LT, Wang H, de Bono M, Wrana JL et al. (2007) Glypican LON-2 is a conserved negative regulator of BMP-like signaling in Caenorhabditis elegans. Curr Biol 17: 159-164. doi: 10.1016/j.cub.2006.11.065. PubMed: 17240342

51. Kinnunen T, Huang Z, Townsend J, Gatdula MM, Brown JR et al. (2005) Heparan 2-O-sulfotransferase, $h s t-2$, is essential for normal cell migration in Caenorhabditis elegans. Proc Natl Acad Sci U S A 102: 1507-1512. doi:10.1073/pnas.0401591102. PubMed: 15671174

52. Stringham EG, Schmidt KL (2009) Navigating the cell: UNC-53 and the navigators, a family of cytoskeletal regulators with multiple roles in cell migration, outgrowth and trafficking. Cell Adh Migr 3: 342-346. doi: 10.4161/cam.3.4.9451. PubMed: 19684480.

53. Stringham E, Pujol N, Vandekerckhove J, Bogaert T (2002) unc-53 controls longitudinal migration in C. elegans. Development 129: 3367-3379. PubMed: 12091307.

54. Fox AN, Zinn K (2005) The heparan sulfate proteoglycan Syndecan is an in vivo ligand for the Drosophila LAR receptor tyrosine phosphatase. Curr Biol 15: 1701-1711. doi:10.1016/j.cub.2005.08.035. PubMed: 16213816 .
55. Johnson KG, Tenney AP, Ghose A, Duckworth AM, Higashi ME et al. (2006) The HSPGs Syndecan and Dallylike bind the receptor phosphatase LAR and exert distinct effects on synaptic development. Neuron 49: 517-531. doi:10.1016/j.neuron.2006.01.026. PubMed: 16476662.

56. Stryker E, Johnson KG (2007) LAR, liprin alpha and the regulation of active zone morphogenesis. J Cell Sci 120: 3723-3728. doi:10.1242/ jcs.03491. PubMed: 17959628.

57. Wacker I, Schwarz V, Hedgecock EM, Hutter H (2003) zag-1, a Znfinger homeodomain transcription factor controlling neuronal differentiation and axon outgrowth in C. elegans. Development 130: 3795-3805. doi:10.1242/dev.00570. PubMed: 12835395.

58. Steven R, Kubiseski TJ, Zheng H, Kulkarni S, Mancillas J et al. (1998) UNC-73 activates the Rac GTPase and is required for cell and growth cone migrations in C. elegans. Cell 92: 785-795. doi:10.1016/ S0092-8674(00)81406-3. PubMed: 9529254.

59. Dudley NR, Labbé JC, Goldstein B (2002) Using RNA interference to identify genes required for RNA interference. Proc Natl Acad Sci U S A 99: 4191-4196. doi:10.1073/pnas.062605199. PubMed: 11904378.

60. Lehner B, Calixto A, Crombie C, Tischler J, Fortunato A et al. (2006) Loss of LIN-35, the Caenorhabditis elegans ortholog of the tumor suppressor p105Rb, results in enhanced RNA interference. Genome Biol 7: R4. doi:10.1186/gb-2006-7-1-r4. PubMed: 16507136.

61. Chaplin T, Ayton P, Bernard OA, Saha V, Della Valle V et al. (1995) A novel class of zinc finger/leucine zipper genes identified from the molecular cloning of the $t(10 ; 11)$ translocation in acute leukemia. Blood 85: 1435-1441. PubMed: 7888665

62. Chinnam M, Goodrich DW (2011) RB1, development, and cancer. Curr Top Dev Biol 94: 129-169. doi:10.1016/B978-0-12-380916-2.00005-X. PubMed: 21295686

63. Morris EJ, Dyson NJ (2001) Retinoblastoma protein partners. Adv Cancer Res 82: 1-54. doi:10.1016/S0065-230X(01)82001-7. PubMed: 11447760.

64. Fay DS, Yochem J (2007) The SynMuv genes of Caenorhabditis elegans in vulval development and beyond. Dev Biol 306: 1-9. doi: 10.1016/j.ydbio.2007.03.016. PubMed: 17434473.

65. Hao JC, Yu TW, Fujisawa K, Culotti JG, Gengyo-Ando K et al. (2001) C. elegans slit acts in midline, dorsal-ventral, and anterior-posterior guidance via the SAX-3/Robo receptor. Neuron 32: 25-38. doi:10.1016/ S0896-6273(01)00448-2. PubMed: 11604136

66. Yu TW, Hao JC, Lim W, Tessier-Lavigne M, Bargmann Cl (2002) Shared receptors in axon guidance: SAX-3/Robo signals via UNC-34/ Enabled and a Netrin-independent UNC-40/DCC function. Nat Neurosci 5: 1147-1154. doi:10.1038/nn956. PubMed: 12379860.

67. Wang D, Kennedy S, Conte D Jr, Kim JK, Gabel HW et al. (2005) Somatic misexpression of germline $P$ granules and enhanced RNA interference in retinoblastoma pathway mutants. Nature 436: 593-597. doi:10.1038/nature04010. PubMed: 16049496.

68. Meyer BJ, Casson LP (1986) Caenorhabditis elegans compensates for the difference in $\mathrm{X}$ chromosome dosage between the sexes by regulating transcript levels. Cell 47: 871-881. doi: 10.1016/0092-8674(86)90802-0. PubMed: 3779843

69. Grishok A, Hoersch S, Sharp PA (2008) RNA interference and retinoblastoma-related genes are required for repression of endogenous siRNA targets in Caenorhabditis elegans. Proc Natl Acad Sci U S A 105: 20386-20391. doi:10.1073/pnas.0810589105. PubMed: 19073934. 